\title{
Lightning medicine in South Africa
}

\author{
Ryan Blumenthal, Estelle Trengrove, Ian R Jandrell, Gert Saayman
}

South Africa has a rich history of lightning research; however, research on the clinical and pathological effects and features of lightning-related injury (keraunomedicine or lightning medicine) remains neglected locally. By providing an overview of keraunomedicine and focussing on South African perspectives, we hope to raise awareness and propose that a concerted and co-ordinated attempt be made to report and collate data regarding lightning strike victims in South Africa.

S Afr Med J 2012;102(7):625-626.
At any moment, about 2000 thunderstorms occur worldwide, producing about 100 lightning strikes each second or 8 million strikes each day. Excluding incidental catastrophes and disasters, lightning causes more deaths than any other natural event or phenomenon, ${ }^{1}$ claiming about 24000 lives each year. It is also estimated that some 240000 people a year will survive a lightning strike. ${ }^{2}$

Lightning poses a risk to people, animals, plants and industrial systems. Its damaging characteristics are primarily due to the immense electrical potential differences and currents that are generated. ${ }^{3}$ Global climate change may also affect lightning patterns and intensity; some studies indicate that global warming will increase overall lightning activity. C Price, in a paper presented at the 29th Symposium on Lightning Protection held in Uppsala, Sweden, in June 2008, suggested that atmospheric electrical activity could be used as a global 'thermometer'. The possibility of harnessing the energy derived from lightning activity remains an elusive but interesting driver of research in this field.

Lightning is a complex phenomenon; understanding its effects on humans, animals and plants, and on the built environment, requires knowledge and expertise involving several disciplines, including climatology, engineering and medicine. To make substantial progress in our understanding of the effects of lightning, a more integrated and multidisciplinary research approach is essential.

Certain areas of Southern Africa have a particularly high incidence of lightning and thunderstorm activity. Early maps of lightning ground flash density $(\mathrm{Ng})$ in South Africa were based on data obtained for the period 1975 - 1986. Owing to the nature of the measurement systems, it was not possible to determine the number of strokes per flash.

More recently, the South African Weather Service installed a 20-station Southern African Lightning Detection Network (SALDN) comprising 19 strategically located sensors in South Africa and 1 in Swaziland. The SALDN was commissioned during 2005, and data have been collected since January 2006., ${ }^{4,5}$ Although representing a limited period, SALDN data analysis suggests that the ground flash density values for areas with $\mathrm{Ng}>8$ may have been previously

Department of Forensic Medicine, University of Pretoria Ryan Blumenthal, MB ChB, MMed (Med Forens), Dip For Med (SA), FC For Path (SA) Gert Saayman, MB ChB, MMed (Med Forens), FC For Path (SA)

School of Electrical and Information Engineering, University of the Witwatersrand, Johannesburg

Estelle Trengrove, PrEng, BA, BSc (Eng), MSc, FSAIEE, MIEEE

Ian R Jandrell, PrEng, BSc (Eng), PhD, FSAIEE, MIEEE significantly underestimated. The SALDN identifies a number of areas with $\mathrm{Ng}$ values higher than the maximum value of 14 flashes/ $\mathrm{km}^{2} /$ year previously reported by the Council for Scientific and Industrial Research (CSIR), having detected areas with $\mathrm{Ng}$ values of up to $23 \mathrm{flashes} / \mathrm{km}^{2} /$ year. This significant observation will have an effect on determining the risk posed to systems, living beings and agriculture. Economic losses associated with lightning strikes may be immense - for those involved in game farming, where rare species of animals may be very valuable, and for crop farming and forestry, where lightning-induced fires can cause severe destruction. Information from insurance companies may dovetail well with recent results obtained from lightning research.

The higher Ng values in South Africa happen to coincide with the distribution of its population, with more ground flashes taking place on the Highveld and Escarpment, where there is a higher population density (Fig. 1).

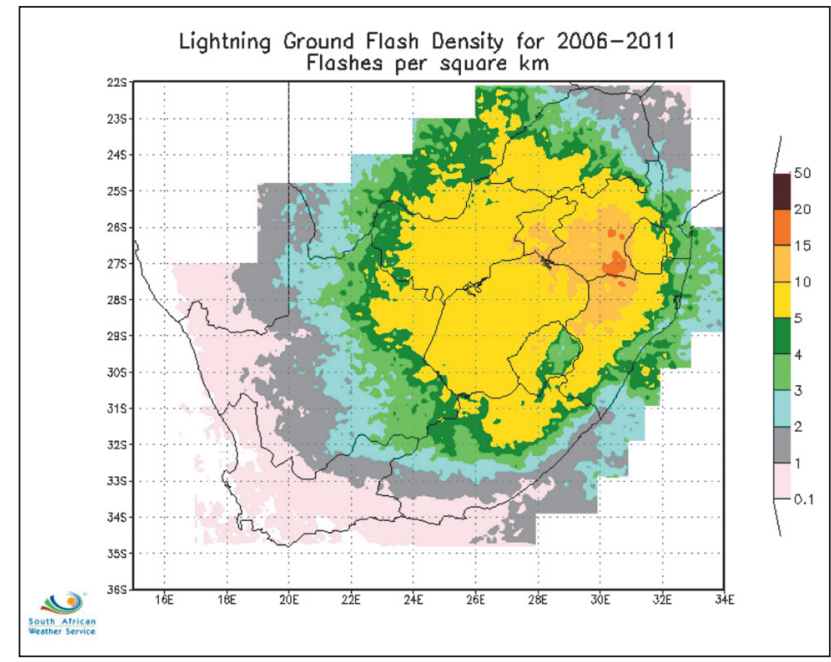

Fig. 1. Lightning ground flash density in South Africa.

South Africa has had a rich tradition of lightning-related research, with Schonland, Malan, Anderson, Eriksson, Proctor and Geldenhuys having been internationally acknowledged in this field. While early work can be traced back to the 1920s, when the first electric field measurements were made, the work of Schonland and Malan (also considered to be the founding members of the CSIR) perhaps was of most value in the early years. The CSIR has maintained its lightning research activities, and from the 1960s has actively participated in the development and testing of lightning detection equipment through the National Electrical Engineering Research Institute (NEERI) in Pretoria, and in collaboration with Cigré (International Council on Large Electric Systems). 


\section{Lightning medicine and related research in South Africa}

Before 1998, almost all reported lightning morbidity and mortality cases emanated from the lay press. In 1998, the National Injury Mortality Surveillance System (NIMSS) was launched in South Africa. Its annual report is intended to capture data from medicolegal mortuaries, and is based on non-natural deaths. This system has provided more reliable data regarding lightning deaths from some centres in South Africa.

Eminent South African electrical engineers, including $\mathrm{R} B$ Anderson, A E Carte, A J Eriksson, I R Jandrell and I S McKechnie, have made significant research contributions to lightning medicine, which in turn has led to greater understanding of the mechanisms of lightning injury in the human body and has played a role in developing lightning safety guidelines and standards for South Africa.

The study of lightning and its effects on humans and animals (keraunomedicine) is rapidly developing, with substantial representation at international conferences on lightning research, trauma and forensic medicine. ${ }^{6}$ Sadly, South Africa has little co-ordinated research, despite the opportunities to engage in this interesting field.

The clinical management of a lightning strike survivor may require a multidisciplinary approach because of its many resulting effects and potential long-term sequelae. Paramedics, emergency medicine physicians, intensive care specialists, neurologists, ENT specialists, speech-and-hearing therapists, occupational therapists, physiotherapists and general practitioners may all be involved, and would require a thorough knowledge of the effects of lightning to effectively contribute to patient management. No standard assessment guidelines and/or management protocols have been published locally, with most patients being managed on an ad hoc basis.

Recent research into lightning mortality data has been undertaken in Gauteng, Transkei and Swaziland, ${ }^{7-9}$ the first retrospective keraunopathology studies for southern Africa. Studies from Gauteng highlight the high number of lightning fatality cases for the region compared with the rest of the world. ${ }^{10}$

According to published data, the death rate due to lightning strike in South Africa varies from 1.5 to 8.8 per million inhabitants per year. In the USA, the reported rate is 0.42 deaths per million people per year (data from 1959 to 1994), ranging from 0.3 to 2.0 per million people. ${ }^{10}$ The incidence of lightning-related fatalities therefore seem to be much higher in South Africa than in the USA. There are up to 100 lightning-related fatalities annually in South Africa, and it is probable that there are at least 4 or 5 times as many survivors of lightning strike presenting for clinical treatment (R Blumenthal unpublished data).

In 2006, published guidelines proposed a more systematic and detailed approach to the medico-legal investigation of deaths apparently related to lightning, including that the scene of death should be visited by pathologists and/or other experts, that injuries be photographed at autopsy, and that tissues, including skin wounds etc., be histologically examined. ${ }^{11,12}$ A more standardised and structured approach would make better data available for future research, including such interesting pathological findings as Lichtenberg figures, 'tip-toe' signs and barotrauma-related phenomena, and more reliable lightning mortality data would become available.
There are virtually no published data on lightning morbidity in South Africa (how many lightning strike survivors, the nature of their symptoms and injuries, etc.). Casualty and emergency medicine units should provide case reports and retrospective reviews of lightning strike survivors. Diverse and interesting clinical effects have been reported in the international literature, which would require further research and evaluation by clinicians and researchers working together to validate or refute largely anecdotal reported findings and long-term complications. Many complications have been attributed to lightning strike, some of which are mythical; individuals and groups have entrenched beliefs that lightning may be directed to strike someone, that significant personality changes ensue after lightning strike, etc. A research project has recently been initiated to understand cultural and traditional South African beliefs regarding lightning.

In a society with rapid urban and rural electrification, forensic pathologists are seeing an increase in fatalities owing to electricity exposure and its association with illegal activities such as cable theft and illicit household electrical connections. ${ }^{12}$ These effects, too, require study and reporting, and could be funded by stakeholders such as local authorities and government agencies, Eskom, and shortterm insurance companies.

\section{Conclusions}

We propose that consideration be given to establishing a multidisciplinary lightning/electricity interest group involving relevant scientific disciplines (including clinicians) and which may co-ordinate and facilitate research and funding in this field. This group could develop standard assessment guidelines and/or medical management protocols. Recently, such a multidisciplinary interest group was established at the University of the Witwatersrand (Lightning Interest Group for Health, Technology and Science (LIGHTS)). Its intentions are to provide a platform for lightning researchers to share their findings within Africa, to share knowledge on lightning physics and related sciences and foster inter-institution partnerships and collaborations to generate new knowledge, research and treatment opportunities. Interested parties may contact the corresponding author.

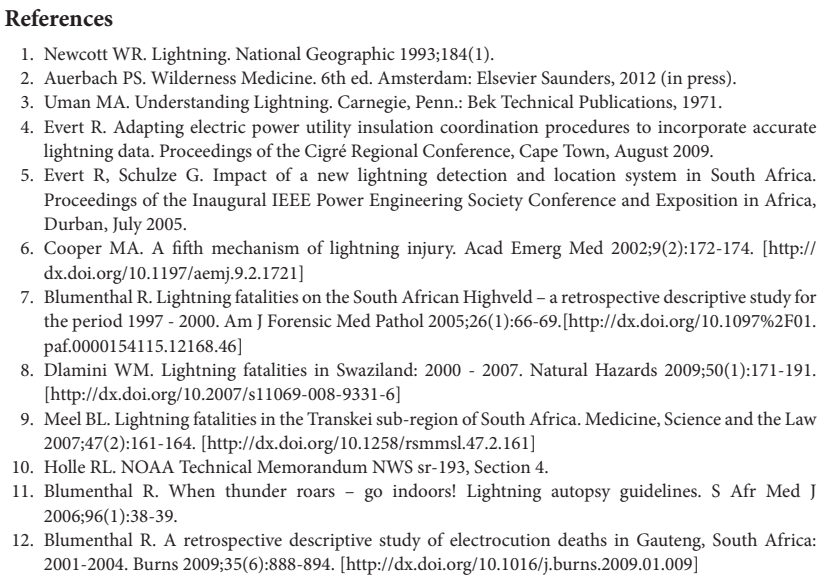

12. Blumenthal R. A retrospective descriptive study of electrocution deaths in Gauteng, South Africa: 2001-2004. Burns 2009;35(6):888-894. [http://dx.doi.org/10.1016/j.burns.2009.01.009] 